The Astrophysical Journal, 201: 90-97, 1975 October 1

(C) 1975. The American Astronomical Society. All rights reserved. Printed in U.S.A.

\title{
MOLECULAR HYDROGEN IN THE GALAXY AND GALACTIC GAMMA RAYS
}

\author{
F. W. STECKER \\ Theoretical Studies Group, NASA Goddard Space Flight Center, Greenbelt \\ P. M. Solomon \\ Department of Earth and Space Sciences, State University of New York, Stony Brook \\ N. Z. SCOVILLE \\ Owens Valley Radio Observatory, California Institute of Technology, Pasadena \\ AND \\ C. E. RYTER* \\ Laboratory for High Energy Astrophysics, NASA Goddard Space Flight Center, Greenbelt \\ Received 1975 February 5; revised 1975 March 26
}

\begin{abstract}
Recent surveys of $2.6-\mathrm{cm} \mathrm{CO}$ emission and $100-\mathrm{MeV} \gamma$-radiation in the galactic plane reveal a striking correlation suggesting that both emissions may be primarily proportional to the line-ofsight column density of $\mathrm{H}_{2}$ in the inner Galaxy. Both the $\gamma$-ray and $\mathrm{CO}$ data suggest a prominent ring or arm consisting of cool clouds of $\mathrm{H}_{2}$ at a galactocentric distance of $\sim 5 \mathrm{kpc}$ with a mean total hydrogen density equivalent to $\sim 5$ atoms $\mathrm{cm}^{-3}$. Estimates are made of column densities of $\mathrm{H}_{2}$ at $l^{\mathrm{II}}=0^{\circ}$. The estimates at $0^{\circ}$ are compared with estimates from infrared and X-ray absorption measurements. These estimates are all consistent and indicate that $\mathrm{H}_{2}$ is far more abundant than $\mathrm{H}_{\mathrm{I}}$ in the inner Galaxy and is the key to a more satisfactory explanation of the $\gamma$-ray observations than previous suggestions. The importance of $\mathrm{H}_{2}$ in understanding galactic $\gamma$-ray observations is also reflected in the correlation of galactic latitude distribution of $\gamma$-rays and dense dust clouds. The deduced cosmic-ray distribution inferred from the calculations is similar to that of galactic supernova remnants, suggesting a galactic origin for most cosmic rays.

A detailed calculation of the $\gamma$-ray flux distribution in the $0^{\circ}-180^{\circ}$ longitude range using the CO data to obtain the average distribution of molecular clouds in the Galaxy shows that most of the enhancement in the inner Galaxy is due to $\pi^{0}$-decay radiation and the 5-kpc ring plays a major role. Detailed agreement with the $\gamma$-ray data is obtained with the additional inclusion of contributions from bremsstrahlung and Compton radiation of secondary electrons and Compton radiation from the intense radiation field near the galactic center.
\end{abstract}

Subject headings: galactic structure — gamma rays — molecular, interstellar

\section{INTRODUCTION}

Molecular hydrogen has long been suspected to be an important component of interstellar gas because it is the most stable low-temperature form of the most abundant element in the Galaxy. A review of the role of molecules in interstellar space has been given by Solomon (1973). Molecular hydrogen is expected to be the predominant form of hydrogen in cool clouds of sufficient density (Solomon and Wickramasinghe 1969; Hollenbach and Salpeter 1971; Hollenbach et al. 1971). However, despite its abundance, it is difficult to measure its galactic distribution directly. The detection of Lyman absorption bands from $\mathrm{H}_{2}$ was reported by Carruthers (1970), and strong absorption lines from interstellar $\mathrm{H}_{2}$ in almost all nearby clouds observed have been seen in the ultraviolet spectra of reddened stars by the Copernicus satellite (Spitzer et al. 1973). However, ultraviolet observations of $\mathrm{H}_{2}$ at distances greater than $1 \mathrm{kpc}$ and in any cloud

* NAS-NRC Post-Doctoral Resident Research Associate; Permanent Address: Centre d'Etudes Nucleaires de Sacaly, 91190 Gif-sur-Yvette, France. with greater than 2 mag of visual extinction are not feasible because of the large extinction of ultraviolet radiation by interstellar dust. Also, because the hydrogen molecule has no permanent dipole moment, no dipole radiation is expected from $\mathrm{H}_{2}$; and quadrupole vibration-rotation features, while potentially detectable in the infrared in dark'clouds, are inherently very weak. The direct ultraviolet observations of Spitzer et al. (1973) have indicated that $\mathrm{H}_{2}$ is a significant component of the interstellar gas; and because the hot young stars that delineate the spiral features of our Galaxy form from cool dense clouds of primarily molecular hydrogen, it is important to determine the large-scale distribution of $\mathrm{H}_{2}$ in the Galaxy in order to understand galactic structure. Results from two promising methods for indirectly studying the galactic distribution of $\mathrm{H}_{2}$ are discussed here, viz., recent galactic surveys of $100-\mathrm{MeV} \gamma$-radiation and $2.6 \mathrm{~mm}$ radio line emission from the $\mathrm{J}=1 \rightarrow 0$ transition of $\mathrm{CO}$ molecules. To these surveys, which reflect the extent and distribution of $\mathrm{H}_{2}$ in the plane of the Galaxy, we will add corroborating information on the amount and latitude distribution of gas in the 


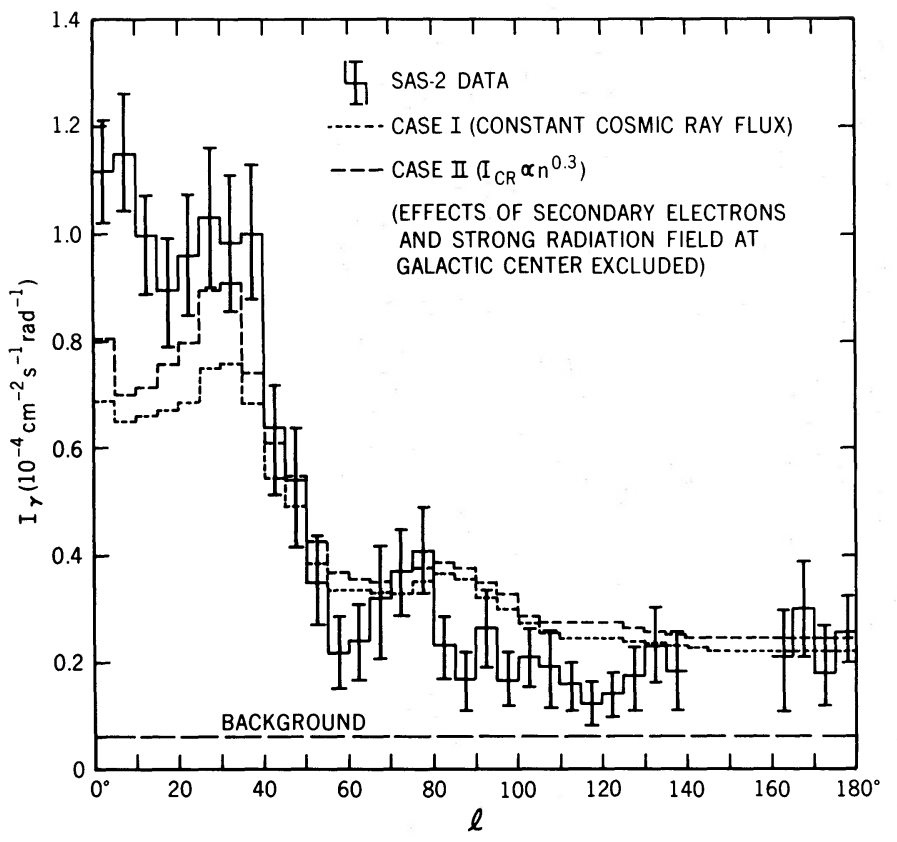

FIG. 1.-Comparison of the results of our numerical calculations with the SAS-2 data of Fichtel et al. (1975) for the two cases discussed in the text and neglecting the contributions from increased secondary electron production and radiation density in the inner Galaxy.

direction of the galactic center supplied by X-ray, optical, and infrared absorption measurements.

\section{THE RECENT SAS-2 GAMMA-RAY GALACTIC LONGITUDE OBSERVATIONS}

Fichtel et al. (1975) have recently reported the results of a sky survey made of $100-\mathrm{MeV} \gamma$-rays using a spark chamber aboard the SAS-2 satellite. Their results on the $\gamma$-ray flux measured within $\pm 10^{\circ}$ of the galactic plane are shown in Figure 1. While it is clear that the general intensity of the flux in the half-plane away from the galactic center can be understood as arising from the decay of $\pi^{0}$ mesons produced in cosmic-ray interactions primarily with atomic hydrogen (based on the production rate calculated by Stecker 1970, 1973), the large intensity-particularly for the $60^{\circ}$ longitude region about the galactic center-cannot be understood so simply. Indeed, the similar galactic longitude distribution observed by the OSO-3 detector was also noted to be distinctly uncorrelated with the $21-\mathrm{cm}$ distribution by Clark et al. (1970). The OSO-3 result implied an increase in cosmic rays, unseen gas, or both in the inner Galaxy, and it was suggested by Stecker (1969, 1971) and Stecher and Stecker (1970) that molecular hydrogen unseen in $21-\mathrm{cm}$ surveys could account for a large part of the $\gamma$-ray enhancement in the inner Galaxy. Black and Fazio (1973) have recently explored the possibility that dark molecular hydrogen clouds are $\gamma$-ray sources. ${ }^{1}$

1 Possible enhancements in the galactic cosmic-ray flux in the inner galaxy were also explored by Ginzburg and Khazan (1972) and Strong et al. (1973). The initially reported SAS-2 result s (Kniffen et al. 1973) were noted by Stecker et al. (1974)
The SAS-2 data obtained by Fichtel et al. (1975) show pronounced peaks near $180^{\circ}$ in the direction of the Crab nebula and near $270^{\circ}$ in the direction of Vela X. These sources can be separated from a discussion of the general diffuse galactic radiation, and they have been discussed by Fichtel et al. (1975). The general trend of the remaining flux observed by SAS-2 strengthens the case made by the earlier OSO-3 data that neither the distribution nor the absolute flux of $\gamma$-radiation can be explained based on the $21-\mathrm{cm}$ data. A model has been proposed by Bignami and Fichtel (1974) and Bignami et al. (1975) based on producing large enhancements at the locations of arms mapped by $21-\mathrm{cm}$ surveys by postulating higher gas densities than are seen in $21-\mathrm{cm}$ and proportionately higher cosmic-ray intensities in the arms. However, Burton et al. (1975) indicate that the arms outside of $25^{\circ}$ from the galactic center are optically thin in $21 \mathrm{~cm}$, and significantly higher amounts of $\mathrm{H} \mathrm{I}$ in the arms than those deduced in previous surveys appear to be ruled out. We therefore feel that the galactic $\gamma$-ray observations can be better understood by using other observations in addition to $21-\mathrm{cm}$ surveys to determine the role of $\mathrm{H}_{2}$ clouds invisible in 21-cm emission.

and Puget and Stecker (1974) to imply a particularly pronounced enhancement in the $\gamma$-ray emissivity in a toroidal region about $5 \mathrm{kpc}$ from the galactic center, and a model was suggested by them for enhancing the galactic cosmic-ray flux in that region by first-order Fermi acceleration. Possible correlations with the galactic magnetic field strength were recently studied by Schlickeiser and Thielheim (1974). Other discussions of the origin of galactic $\gamma$-rays have been given by Dodds et al. (1974) and by Cowsik and Voges and by Paul, Casse, and Cesarsky in the same proceedings. For further discussion see the recent review by Stecker (1975a). 


\section{THE GALACTIC CO DISTRIBUTION AND THE} MOLECULAR RING AT $\sim 5$ KILOPARSECS

A survey of the galactic longitude distribution of $\mathrm{CO}$ emission in the galactic plane has recently been made by Scoville and Solomon (1975). The importance of this survey in understanding the distribution of $\mathrm{H}_{2}$ in the Galaxy lies in the fact that $\mathrm{CO}$ is an excellent tracer of $\mathrm{H}_{2}$. The relationship between $\mathrm{H}_{2}$ and $\mathrm{CO}$ in molecular clouds, based on the fact that the most important source of $\mathrm{CO}$ excitation in these clouds is by collisions with $\mathrm{H}_{2}$ and radiative trapping, has recently been discussed by Scoville and Solomon (1974) and Goldreich and Kwan (1974).

Scoville and Solomon (1975) have used the velocity profile data obtained in their $\mathrm{CO}$ survey in conjunction with the Schmidt (1965) rotational model of the Galaxy to determine the mean distribution of $\mathrm{CO}$ in the Galaxy as a function of galactocentric distance for distances greater than $2.6 \mathrm{kpc}$. This distribution shows a broad peak with a maximum near $5 \mathrm{kpc}$ which they have concluded indicates a ring of $\mathrm{H}_{2}$ clouds in this region. The general form of the molecular cloud distribution obtained by Scoville and Solomon has recently been confirmed in an independent CO survey by Burton et al. (1975). The connection between this feature and the $\gamma$-ray emission ring at $\sim 5 \mathrm{kpc}$ (Puget and Stecker $1974^{2}$ ) led to the suggestion that the $\gamma$-ray data also provide evidence for the molecular cloud ring near $5 \mathrm{kpc}$ (Solomon and Stecker 1974). Coincidently, there is also a similar distribution and peak in the giant H II regions of the Galaxy (Mezger 1970). This may be understood to be the effect of hot young stars in OB associations being formed out of dense molecular clouds in this ring. The formation of such a prominent molecular ring poses an intriguing problem for galactic structure theory. Such a large-scale region of relatively cool high-density clouds may be the result of a shock front and thus provide a key to understanding the structure of the inner Galaxy (e.g., Roberts 1970; Sanders and Prendergast 1974).

The $\gamma$-ray emissivity measurements from SAS-2 can be used to place an upper limit on the $\mathrm{H}_{2}$ density at 5 kpc. Puget and Stecker (1974) have estimated the emissivity ratio

$$
\frac{Q_{\gamma}(5 \mathrm{kpc})}{Q_{\gamma}(10 \mathrm{kpc})} \approx 11 \pm 2,
$$

which is proportional to the ratio of the product of total baryon density and cosmic-ray intensity at 5 and $10 \mathrm{kpc}$, respectively. Assuming that the ratio of cosmic-ray intensities $I_{\mathrm{CR}}(5 \mathrm{kpc}) / I_{\mathrm{CR}}(10 \mathrm{kpc}) \geq 1$, we find that $n_{\mathrm{H}_{2}}(5 \mathrm{kpc}) \leqslant(5 \pm 1)$ molecules $\mathrm{cm}^{-3}$. This result, however, only includes the statistical errors in the SAS fluxes, and the error in the analysis is probably underestimated. Strong (1975) has obtained a similar result. The CO data (Scoville and Solomon 1975) give an estimated $\mathrm{H}_{2}$ density in the $5 \mathrm{kpc}$ region of between 2 and 7 molecules $\mathrm{cm}^{-3}$. The $\gamma$-ray upper limit is thus

\footnotetext{
${ }^{2}$ Based on the data as given by Kniffen et al. (1973).
}

consistent with the range allowed by the $\mathrm{CO}$ observations.

\section{MOLECULAR HYDROGEN AND TOTAL COLUMN DENSITIES IN THE DIRECTION $l=0^{\circ}$}

By using the distribution of $\mathrm{CO}$ emission (Fig. 4 of Scoville and Solomon 1975) one can estimate the amount of molecular hydrogen at $l=0^{\circ}$ excluding the galactic nuclear region. The result is $3 \times 10^{22} \leq$ $\left\langle 2 N_{\mathrm{H}_{2}}\right\rangle \leq 10^{23} \mathrm{~cm}^{-2}$. This number can also be estimated from measurements of X-ray and infrared absorption measurements in the direction of the galactic nucleus (Ryter et al. 1975).

$\mathrm{X}$-rays and infrared radiation are moderately attenuated when traveling through several $\mathrm{kpc}$ in the Galaxy. The infrared is attenuated by interstellar grains, and the X-rays are attenuated by photoelectric absorption due primarily to carbon, nitrogen, and oxygen. Thus the amount of matter on the line of sight to a source with a known (or calculated) energy spectrum can be deduced subject to the assumption of cosmic abundance ratios.

$\mathrm{X}$-ray absorption in front of the galactic center source, GCX, has been measured by Tucker et al. (1973), and the amount of "equivalent atomic" hydrogen along the line of sight has been given as $\left\langle N_{\mathrm{H}}\right\rangle=(9 \pm 2) \times 10^{22}$ atoms $\mathrm{cm}^{-2}$ based on the cross sections of Brown and Gould (1970) assuming that the universal abundances hold and that all the hydrogen is atomic. However, this value is almost an order of magnitude higher than $\left\langle N_{\mathrm{H}}\right\rangle$ given in the $21-\mathrm{cm}$ surveys, and a strong argument can be made that most of the hydrogen is in molecular form. The ratio of absorption cross sections $\sigma_{\mathrm{H}_{2}} / 2 \sigma_{\mathrm{H}}$ at $3 \mathrm{keV}$ has been given as 7.7 (Brown and Gould 1970), but more recent calculations indicate a value of 1.7 (Kaplan and Markin 1973). Using the smaller value for $\sigma_{\mathrm{H}_{2}}$, which is supported by the recent measurements of Crasemann et al. (1974), results in a negligible increase in the effective absorption rate per $\mathrm{H}$ atom (most absorption being due to $\mathrm{C}, \mathrm{N}, \mathrm{O}$ ), but the larger value increases this rate by $\sim 30$ percent, lowering the total column density estimate to the value $\left\langle 2 N_{\mathrm{H}_{2}}+N_{\mathrm{HI}}\right\rangle=6.7 \times 10^{22} \mathrm{~cm}^{-2}$ (Ryter et al. 1975$)$.

This column density can also be estimated allowing for the possibility that the CNO abundance relative to hydrogen may increase in the direction of the galactic center. Spiral galaxies in general seem to exhibit abundance gradients with higher relative content of CNO in the central region (Peimbert 1968; McClure 1969; Searle 1971; Benvenuti et al. 1973). Taking as typical figures $\mathrm{C}$ and $\mathrm{O}$ enriched by $\sim 1.5$, at $5 \mathrm{kpc}$, using the smaller $\sigma_{\mathrm{H}_{2}}$ (Crasemann et al. 1974), we obtain $\left\langle 2 N_{\mathrm{H}_{2}}+N_{\mathrm{HI}}\right\rangle \approx 6.5 \times 10^{22} \mathrm{~cm}^{-2}$.

Infrared absorption in the line of sight to the galactic center has been measured at $0.35,0.90,1.65,2.2$, and $3.4 \mu$ by Becklin and Neugebauer (1968) and Spinrad et al. (1971). Using a standard reddening curve and postulating a thermal spectrum for the infrared source at the galactic center, a color excess of $E_{B-V}=9.5$ 
TABLE 1

Column Densities of Hydrogen at $l=0^{\circ}$ Excluding the Galactic Nucleus

$\left(\times 10^{-22}\right)\left(\mathrm{cm}^{-2}\right)\left(N_{\text {G.C., } \odot}\right)$

\begin{tabular}{|c|c|c|}
\hline$\left\langle N_{\mathrm{HI}}\right\rangle$ from $21 \mathrm{~cm}$ radio $\ldots \ldots \ldots \ldots \ldots \ldots$ & $\begin{array}{l}\geqslant 0.6 \text { to } 1.5 \\
1 \text { to } 2 \\
\approx 1.2\end{array}$ & $\begin{array}{l}\text { Daltabuit and Meyer } 1972 \\
\text { Kerr and Westerhout } 1965 \\
\text { Clark } 1965\end{array}$ \\
\hline$\left\langle 2 N_{\mathrm{H}_{2}}\right\rangle$ from $\mathrm{CO} \ldots \ldots \ldots \ldots \ldots \ldots \ldots$ & 3 to 10 & Scoville and Solomon 1975 \\
\hline $\begin{array}{l}\left\langle 2 N_{\mathrm{H}_{2}}+N_{\mathrm{H}}\right\rangle \text { from SAS-2 } \gamma \text {-ray flux....... } \\
\left\langle 2 N_{\mathrm{H}_{2}}+N_{\mathrm{H}}\right\rangle\end{array}$ & $\begin{array}{l}\lesssim(11.5 \pm 2) \\
6.5 \text { to } 9\end{array}$ & $\begin{array}{l}\text { This work }\left(I_{\mathrm{CR}} \geqslant I_{\odot}\right) \\
\sigma_{\mathrm{H}_{2}} / 2 \sigma_{\mathrm{HI}} \leq 1.3(\text { Kaplan and Markin 1973; Crasemann et al. } \\
\quad 1974)\end{array}$ \\
\hline$\left\langle 2 N_{\mathrm{H}_{2}}+N_{\mathrm{H}}\right\rangle$ from infrared absorption.... & 5 to 7.5 & Ryter et al. 1975 \\
\hline
\end{tabular}

$(+2,-1)$ mag is deduced with an implied visual extinction $A_{V}=29 \mathrm{mag}$. Using the relation

$$
N_{\mathrm{H}} \approx 7 \times 10^{21} E_{B-V} \mathrm{~cm}^{-2}
$$

(Jenkins and Savage 1974; Gorenstein 1975; Ryter et al. 1975), the amount of matter on the line of sight to the galactic center is estimated to be $5 \times 10^{22} \leq$ $\left\langle N_{\mathrm{HI}}+2 N_{\mathrm{H}_{2}}\right\rangle \leq 7.5 \times 10^{22} \mathrm{~cm}^{-2}$.

All of the above methods give consistent results which are summarized in Table 1.

Scoville et al. (1974) estimate the total mass of the molecular disk near the galactic center to be

$$
4 \times 10^{7} \leqslant\left(M_{\mathrm{GC}} / M_{\odot}\right) \leqslant 10^{8} .
$$

This mass is contained in massive clouds in a ringlike structure about $200 \mathrm{pc}$ from the galactic center. There does not appear to be any massive cloud situated exactly in the line of sight to Sgr A, and this feature may therefore not contribute to the infrared or X-ray absorption.

The molecular disk would yield a contribution to the measured $\gamma$-ray flux at $l=0^{\circ}$ of

$$
\begin{aligned}
& \Delta I_{\gamma, \mathrm{GC}} \\
& \quad=\frac{1}{\Delta l}\left(1.3 \times 10^{-6}\right) R_{\mathrm{pc}}{ }^{-2}\left(M_{\mathrm{GC}} / M_{\odot}\right) \mathrm{cm}^{-2} \mathrm{~s}^{-1} \mathrm{rad}^{-1},
\end{aligned}
$$

assuming $I_{\mathrm{CR}}\left(\mathrm{GAL} . \mathrm{CEN}\right.$ ) $=I_{\mathrm{CR}}(10 \mathrm{kpc})$ (Ginzburg and Khazan 1972; Stecker 1973; Black and Fazio 1974). This yields an estimated flux in the range

$$
0.6 \times 10^{-5} \leqslant \Delta I_{\gamma, \mathrm{GC}} \leqslant 1.5 \times 10^{-5} \mathrm{~cm}^{-2} \mathrm{~s}^{-1} \mathrm{rad}^{-1},
$$

which is only about one-tenth of the observed flux at $l=0^{\circ}$.

\section{GALACTIC GAMMA-RAY FLUX AS A FUNCTION OF LONGITUDE}

\section{a) Calculations}

In performing numerical calculations of the longitude distribution of the galactic $\gamma$-ray flux from $\pi^{0}$ decay, we have used the survey of Scoville and Solomon (1975) to obtain the relative distribution of molecular hydrogen in the Galaxy as a function of galactocentric distance $\varpi$ and have normalized to a total column density in the direction of the galactic center using the X-ray and infrared absorption measurements (Table 1) which are consistent with, but presumably more accurate than, the column densities deduced from the $\gamma$-ray or $\mathrm{CO}$ results. The contribution from atomic hydrogen was estimated based on the numbers given by Kerr and Westerhout (1965) and Westerhout (1970). The $\gamma$-ray longitude distribution is given by the relation

$$
\begin{aligned}
& I_{\gamma}(l) \\
& \quad=\frac{q_{\odot}}{4 \pi} \int_{-10^{\circ}}^{+10^{\circ}} d b \int_{0}^{h \cot b} d s\left(\frac{J(\varpi)}{J_{\odot}}\right)\left[n_{\mathrm{HI}}(\varpi)+2 n_{\mathrm{H}_{2}}(\varpi)\right],
\end{aligned}
$$

where $b$ is galactic latitude, $h$ is the half-thickness of the galactic plane taken to be $110 \mathrm{pc}$ in the inner Galaxy, ${ }^{3} q_{\odot}=1.3 \times 10^{-25} \mathrm{~s}^{-1}$ is the specific $\gamma$-ray luminosity from $\pi^{0}$ decay, electron bremsstrahlung, and Compton radiation above $100 \mathrm{MeV}$ in the solar galactic neighborhood, ${ }^{4} s$ is heliocentric distance, and

$$
\varpi=\left(\varpi_{\odot}^{2}+s^{2}-2 \varpi_{\odot} s \cos l\right)^{1 / 2} .
$$

The quantity $J_{\odot}$ is the cosmic-ray intensity in the local galactic neighborhood, and $J(\varpi)$ is the cosmic-ray intensity assumed to be a function of galactocentric distance. The quantity $n_{\mathrm{H}}(\varpi)$ is the neutral hydrogen density obtained from $21-\mathrm{cm}$ measurements, and $n_{\mathrm{H}_{2}}(\varpi)$ is the density of $\mathrm{H}_{2}$ inferred from the $\mathrm{CO}$ survey normalized using the data from Table 1 so that

$$
\begin{aligned}
N_{\mathrm{GC}, \odot} & \equiv\left(2 N_{\mathrm{H}_{2}}+N_{\mathrm{HI}}\right)_{\mathrm{GC}, \odot} \\
& =\int_{0}^{\varpi \odot} d \varpi\left[n_{\mathrm{HI}}(\varpi)+2 n_{\mathrm{H}_{2}}(\varpi)\right] \\
& =(7 \pm 1.5) \times 10^{22} \mathrm{~cm}^{-2} .
\end{aligned}
$$

For the purpose of the calculations to estimate the

\footnotetext{
${ }^{3}$ Allowance for variation of $h$, particularly outside $10 \mathrm{kpc}$ was made by adjusting the values of $n(\varpi)$. The scale height of the molecular clouds is probably somewhat smaller than the value given for the diffuse $\mathrm{H}$ I component adopted here. The error introduced is of the same order as the error in $q_{\odot}$ and $N_{\text {GC, } \odot \text {. }}$

${ }^{4}$ Above $100 \mathrm{MeV}, q_{\pi^{0}} \equiv 1.1 \times 10^{-25} \mathrm{~s}^{-1}$ (Stecker 1970), $q_{\text {brems }} \approx 0.1 \times 10^{-25} \mathrm{~s}^{-1}$ (Stecker 1971), and $q_{\text {Compton }} \approx 0.06 \times$ $10^{-25} \mathrm{~s}^{-1}$ (Stecker 1975a). In the outer Galaxy, the starlight gradient is assumed to vary like the total gas gradient, an approximation which does not affect the calculations very much as long as Compton radiation is such a minor component of the total flux.
} 
effect of cosmic-ray enhancements in the Galaxy, it was assumed that such enhancements may be correlated with the gas distribution so that

$$
\frac{J(\varpi)}{J_{\odot}}=\left[\frac{n_{\mathrm{HI}}(\varpi)+2 n_{\mathrm{H}_{2}}(\varpi)}{n_{\mathrm{HI}, \odot}+2 n_{\mathrm{H}_{2}, \odot}}\right]^{\alpha},
$$

where, from dynamical considerations, the exponent $\alpha \leq 1$ (Parker 1969). ${ }^{5}$

We have calculated $I_{\gamma}(l)$ for two cases. In case I a constant cosmic-ray flux in the Galaxy was assumed $(\alpha=0)$ in order to determine the effect of gas density contrast alone on the $\gamma$-ray longitude distribution. For this case, a value of $N_{\mathrm{Gc}, \odot}=8.5 \times 10^{22} \mathrm{~cm}^{-2}$ was taken, corresponding to the upper limit on the total column density implied by the infrared and X-ray absorption measurements. In case II, it was assumed that $N_{\mathrm{GC}, \odot}=7 \times 10^{22} \mathrm{~cm}^{-2}$ and $\alpha=0.3$, corresponding to an increase of about a factor of 2 in the cosmic-ray flux in the $5-\mathrm{kpc}$ region as indicated by studies of the supernova remnant distribution in the Galaxy (Ilovaisky and Lequeux 1972; Kodaira 1974), scale height measurements (Jackson and Kellman 1974), and synchrotron radiation measurements (Webber 1968; Price 1974; Daniel and Stephens 1975). We consider case II to be the situation most consistent with all the measurements we have to date on the total gas distribution and cosmic-ray distribution in the inner Galaxy. It corresponds to a mean value for the total gas density of $\sim 1$ atom $\mathrm{cm}^{-3}$ at $10 \mathrm{kpc}$ of which $\sim 40$ percent is in molecular form. This agrees with the values given by Spitzer et al. (1973) from measurements of rotational ultraviolet absorption lines of $\mathrm{H}_{2}$ and those given by Jenkins and Savage (1974) for Lyman $\alpha$ lines of $\mathrm{H}$ I. In the 5-kpc region, case II corresponds to a volume-averaged total density of $\sim 5$ atoms $\mathrm{cm}^{-3}$ of which $\sim 80$ percent would be in molecular form.

\section{b) Results}

The results of the numerical calculations for cases I and II are shown in Figure 1 together with the flux distribution given by Fichtel et al. (1975) from the SAS-2 observations for the half-plane from $0^{\circ}$ to $180^{\circ}$ over which the CO measurements of Scoville and Solomon (1975) can be applied. These results indicate that most of the observed $\gamma$-ray enhancement at low longitudes is primarily a result of increased gas density and that the molecular ring near $5 \mathrm{kpc}$ plays an important role in accounting for this increase.

Cosmic rays are limited to a small variation over the galactic disk given by $0.2 \leqslant \alpha \leqslant 0.5$ as derived from the limits on the amount and distribution of gas implied by the observations discussed in $\S$ IV. We thus conclude that cosmic rays cannot vary linearly with gas density over all segments of the Galaxy, nor do they appear to be uniformly distributed.

${ }^{5}$ Bignami et al. (1975), using a different gas distribution, have assumed $\alpha=1$ corresponding to cosmic rays varying linearly with gas density.
The large-scale distribution of cosmic rays in the Galaxy deduced using the observed $\gamma$-ray emissivity distribution and the observed interstellar gas distribution (including that of molecular clouds) is identical to the distribution of supernova remnants in the Galaxy (Kodaira 1974) to within the errors in the observations. Both distributions peak in the $5-\mathrm{kpc}$ region at about twice the total value at $10 \mathrm{kpc}$. The low values of the $\gamma$-ray flux observed in the outer Galaxy indicate that the cosmic-ray flux in the outer Galaxy must be dropping off much faster than the atomic hydrogen observed in $21 \mathrm{~cm}$, as does the supernova remnant distribution. This dropoff in the cosmic-ray flux in the outer Galaxy, indicated by our analysis of the $\gamma$-ray longitude distribution here, is also supported by recent calculations of the $\gamma$-ray latitude distribution in the anticenter direction (Dodds et al. 1975) and provides strong support for the galactic origin of most cosmic rays in the 1 to $10 \mathrm{GeV}$ energy range. Furthermore, the strong correlation between the deduced cosmic-ray flux and the observed supernova distribution over the whole Galaxy supports the hypothesis that supernovae are the source for most cosmic rays (Ginzburg and Syrovatskii 1963; Stecker $1975 b)$.

Some fluctuations in the observed longitude distribution of $\gamma$-radiation are not reproduced exactly by the calculations. This is due to the fact that the calculations were made using an average gas density as a function of galactocentric distance. The observed fluctuations may be understood as due to "hot spots" from nearby gas clouds (FitzGerald 1968; Black and Fazio 1973), and these are discussed in detail by Puget et al. (in preparation). Weak supernova remnants may also account for some of these fluctuations (Fichtel et al. 1975).

The results shown in Figure 1 indicate that the character of the longitude distribution of galactic $\gamma$ radiation corresponds well with the overall density distribution in the Galaxy implied by the $\mathrm{CO}$ and 21 $\mathrm{cm}$ measurements and that this distribution has a broad maximum in the 5 to $6 \mathrm{kpc}$ region. Higher frequency modulations by spiral arms do not appear to us to play a significant role in determining the galactic $\gamma$-ray distribution within the statistical errors and $5^{\circ}$ resolution of the SAS-2 data, at least for the half-plane analyzed here using the $\mathrm{CO}$ data. In the other half-plane, $180^{\circ} \leq l \leq 360^{\circ}$, three apparently sharp features have been identified by Fichtel et al. (1975) with the Scutum, Norma, and " $3 \mathrm{kpc"} \mathrm{arm}$ features designated in $21-\mathrm{cm}$ surveys. The two inner features at $330^{\circ}-335^{\circ}$ and $340^{\circ}-345^{\circ}$ may correspond to a bifurcation of the broad molecular ring near $5 \mathrm{kpc}$ on the other half-plane of the Galaxy; the feature at $310^{\circ}-315^{\circ}$ is perhaps somewhat more puzzling since this should be associated with a correspondingly strong feature at $l \approx 50^{\circ}$ which appears to be absent in both the $\gamma$-ray and CO data. There are, however, large error bars associated with the $310^{\circ}-315^{\circ}$ observation due to the fact that the SAS-2 spark-chamber telescope only viewed this direction obliquely. Also, Puget et al. (in preparation) have indicated that large 
corrections due to nearby features are necessary in the $310^{\circ}-360^{\circ}$ region. Future $\mathrm{CO}$ observations from the southern hemisphere could help increase our understanding of the matter distribution in this region.

Thus, the galactic gas seems to have a large-scale superstructure modulated by spiral arm perturbations similar to that seen in M31 in 21-cm emission (Guibert 1974; Emerson 1974) and in our own Galaxy in nonthermal radio emission (Price 1974), and it appears to be this superstructure which determines the character of the general central enhancement in the $\gamma$-ray longitude distribution. There remains a curious puzzle with regard to the maximum in the interstellar gas density which appears in the 5 to $6 \mathrm{kpc}$ region. Whereas $21-\mathrm{cm}$ measurements in the Galaxy do not show such a clear maximum (e.g., Kerr and Westerhout 1965), the recent high-resolution 21-cm surveys of M31 (Guibert 1974; Emerson 1974) and M81 (Gottesman and Weliachew 1975) do show ringlike features similar to that observed for $\mathrm{CO}$ in the Galaxy. Although the $\mathrm{H}$ I rings in M31 and M81 appear to be broader than the molecular ring observed in $\mathrm{CO}$ in the Galaxy, the $\mathrm{H}$ II region distributionparticularly that in $\mathrm{M} 81$-is more narrowly peaked than the $\mathrm{H}$ I distribution and, as in our Galaxy, the $H$ II region distribution should be better correlated with the molecular cloud distribution. This is particularly obvious in Figure 14 of Gottesman and Weliachew (1975).

Thus, it would appear that in both external galaxies and our own Galaxy the density gradient of the $\mathrm{H}_{2}$ distribution is steeper than that of the $\mathrm{H}$ I distribution, resulting in a decrease in the ratio $n_{\mathrm{H}_{2}} / n_{\mathrm{HI}}$ with distance outward from the region of maximum density.

\section{c) Secondary Electrons and Compton Radiation}

The calculations shown in Figure 1 deviate from the observations for $l<40^{\circ}$. This is due to the fact that the effect of increased production of secondary electrons and positrons, particularly in the 5-6 kpc region, and the effect of high radiation fields in producing Compton radiation in the galactic nuclear region have not been included. Secondary electrons make up approximately 17 percent of the total electron flux above $100 \mathrm{MeV}$ locally (Daniel and Stephens 1975), but their production rate goes up by an order of magnitude in the $5-6 \mathrm{kpc}$ region because it scales like the $\gamma$-ray production rate, both processes being the result of cosmic-ray $\pi$-meson decay. When this effect is included in the calculations and the effect of the enhanced radiation field in producing Compton radiation in the galactic nuclear region is also taken into account, based on the estimates of Dodds et al. (1974), the total flux distribution shown in Figure 2 is obtained. This distribution is compared in Figure 2 with the range of the measurements obtained from SAS-2. The agreement between the calculations and the observations can be seen to be excellent, except perhaps at the higher longitudes where the cosmic-ray flux appears to drop faster than assumed here (but consistent with supernova origin, see previous discussion and Stecker $1975 b$ ). It can also be seen that the total contribution from bremsstrahlung and Compton interactions of both primary and secondary electrons to the galactic $\gamma$-ray flux in the central region is of the order of 30 percent, in agreement with the estimates made by Stecker et al. (1974) using the observed $\gamma$-ray energy spectrum obtained by SAS- 2 .

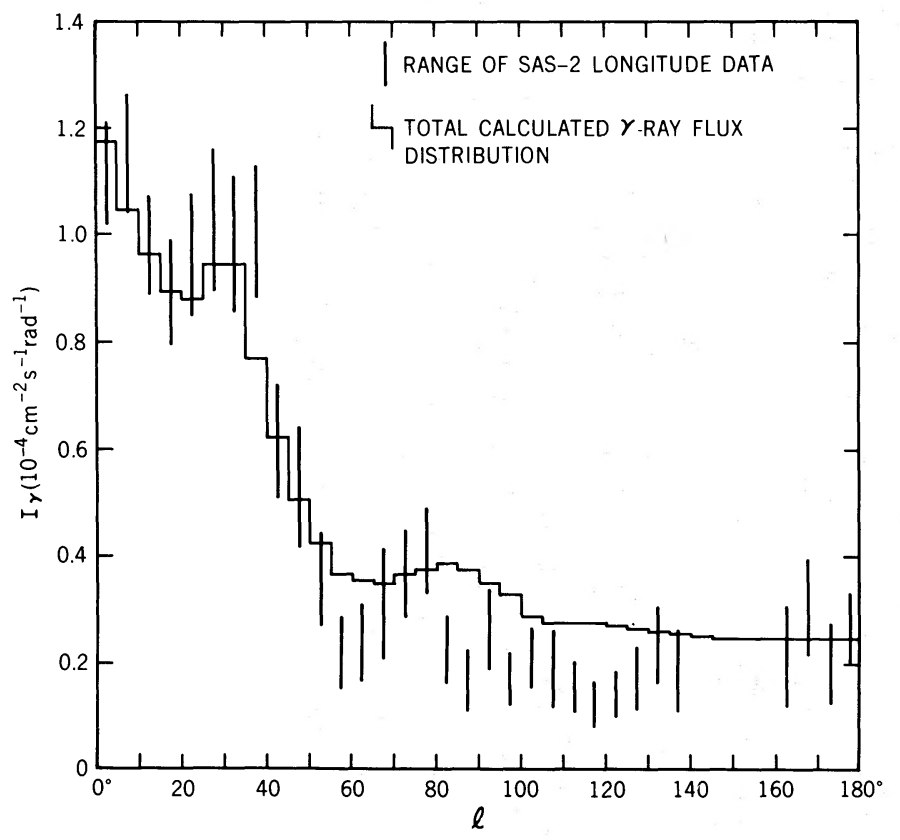

FIG. 2.-Comparison of the total calculated $\gamma$-ray flux distribution with the SAS-2 longitude data. Contributions from increased secondary electron production and enhanced radiation fields in the inner Galaxy are included. 
VI. GAMMA-RAY LATITUDE DISTRIBUTION AND ASSOCIATED LINE-OF-SIGHT REDDENING

In addition to the $\gamma$-ray longitude distribution measurements reported by Fichtel et al. (1975), a galactic latitude distribution was also obtained. This distribution shows an asymmetry with respect to the galactic plane with more flux coming from positive galactic latitudes in the case of moderate latitudes $6^{\circ} \leq|b| \leq 30^{\circ}$. The $21-\mathrm{cm}$ measurements of neutral hydrogen summarized by Daltabuit and Meyer (1972) show less asymmetry of this kind. Thus here, as in the case of the longitude distributions, no detailed correlation is evident between the $\mathrm{H}$ I density and the $\gamma$-ray flux. However, another correlation does present itself.

Recent studies of the reddening of globular clusters by Kron and Guetter (1973) show that clusters at negative latitudes are less reddened than those at positive latitudes. Thus, the observed $\gamma$-ray flux asymmetry in the center direction appears to be associated with the fact that there is a greater number of dense clouds containing dust at positive latitudes than at negative ones in that direction. This is also borne out in the survey of Knapp and Kerr (1974). Such reddening may be associated with the location of Gould's extinction belt (Lynds 1962; Heiles and Jenkins 1975) and a possible correlation between moderate-latitude $\gamma$-rays and Gould's belt was noted by Fichtel et al. (1975) in their discussion of the $\gamma$ ray latitude distribution. All of this can be understood in the context of $\S I V$, where it was pointed out that ultraviolet, X-ray, and infrared absorption measurements indicate that the total column density of gas is proportional to the reddening in a given direction and that in the direction of highly reddened objects most of the hydrogen may well be in molecular form, as expected in dense dust clouds and as is indicated by only a partial correlation with $21-\mathrm{cm}$ emission. The data of Knapp and Kerr (1974) in the region of the sky located between $l=345^{\circ}$ and $30^{\circ}$ and between $b=10^{\circ}$ and $30^{\circ}$ can be used to evaluate the average reddening and $\mathrm{H}$ I column density at $b=20^{\circ}$. We obtain $\left\langle E_{B-V}\left(20^{\circ}\right)\right\rangle=0.26 \mathrm{mag}$ and $\left\langle N_{\mathrm{H}}\left(20^{\circ}\right)\right\rangle=9.7 \times 10^{20}$ $\mathrm{H}$ atoms $\mathrm{cm}^{-2}$. Postulating the same cosmic-ray density as observed locally, we find from the reddening and relation (2) a $\gamma$-ray flux of $2.2 \times 10^{-5} \mathrm{~cm}^{-2} \mathrm{~s}^{-1}$ $\mathrm{sr}^{-1}$ which compares favorably with the flux reported by Fichtel et al. (1975) observed at $b=20^{\circ}$ of 2 to $2.5 \times 10^{-5} \mathrm{~cm}^{-2} \mathrm{~s}^{-1} \mathrm{sr}^{-1}$. However, the flux estimated from the $\mathrm{H}$ I column density is only $1.4 \times$ $10^{-5} \mathrm{~cm}^{-2} \mathrm{~s}^{-1} \mathrm{sr}^{-1}$, which is particularly significant in this case because at high galactic latitudes the $21-\mathrm{cm}$ emission line is not optically thick (Knapp and Kerr 1974). Thus, the situation with regard to the latitude distribution of galactic $\gamma$-rays is analogous to that of the longitude distribution and can be better understood by taking account of $\mathrm{H}_{2}$ unseen in $21-\mathrm{cm}$ surveys of $\mathrm{H}$ I gas.

\section{SUMMARY}

We have shown how the galactic $\gamma$-ray emission, which is proportional to the total density of gas in the Galaxy, is correlated with the galactic CO distribution, $\mathrm{CO}$ being a tracer of interstellar $\mathrm{H}_{2}$. We have also shown that ultraviolet, X-ray, and infrared absorption measurements, which also appear to be correlated with total interstellar gas density, indicate, along with the $\gamma$-ray measurements and $\mathrm{CO}$ measurements, that there are large amounts of $\mathrm{H}_{2}$ in dense clouds in the inner $7 \mathrm{kpc}$ of the Galaxy much in excess of the $\mathrm{H} \mathrm{I}$ seen in 21-cm surveys. Thus, in the inner Galaxy, 21$\mathrm{cm}$ surveys do not appear to give a true indication of the amount and distribution of interstellar hydrogen because they do not indicate the presence of a large amount of $\mathrm{H}_{2}$ which has a different distribution from $\mathrm{H}$ I. The correlation between the galactic latitude distributions of dense clouds (predominantly $\mathrm{H}_{2}$ ) and $\gamma$-ray emission also bear this out. We thus arrive at a more satisfactory explanation of the galactic $\gamma$-ray emission than those suggested previously because it is based upon a wide range of empirical data involving the whole range of the electromagnetic spectrum from radio to $\gamma$-ray wavelengths and thus minimizes model building with arbitrary assumptions. The consistent picture which emerges highlights the significant role of $\mathrm{H}_{2}$ in understanding galactic structure and emphasizes the importance of the knowledge to be gained by future advances in galactic $\gamma$-ray surveys and $\mathrm{CO}$ surveys in understanding galactic dynamics. A particular feature discussed here is the existence of a prominent ring or arm of $\mathrm{H}_{2}$ clouds at $\sim 5 \mathrm{kpc}$. The fact that this region corresponds to one of maximal amounts of $\mathrm{H}$ II in the Galaxy suggests that this ring of dense clouds is the site of most active star formation.

The deduced distribution of cosmic rays inferred from the calculations is similar to that of galactic supernova remnants, peaking in the $5-\mathrm{kpc}$ region at about twice the local value and dropping off in the outer Galaxy, supporting the galactic supernova hypothesis for the origin of most cosmic rays. This point is pursued further elsewhere (Stecker 1975b).

We wish to acknowledge and thank Dr. Jean-Loup Puget, Dr. Giovanni Bignami, and Dr. Giovanni Fazio for stimulating and helpful discussions, Dr. Carl Fichtel for communicating the results of his SAS-2 observations prior to publication, and especially Mr. Joseph Bredekamp for programming the numerical calculations used in this paper.

\section{REFERENCES}

Becklin, E. E., and Neugebauer, G. 1968, Ap. J., 151, 145.

Benvenuti, P., D'Odorico, S., and Peimbert, M. 1973, Astr. and $A p ., 28,447$.
Bignami, G. F., and Fichtel, C. E. 1974, Ap. J. (Letters), 189, L65.

Bignami, G. F., Fichtel, C. E., Kniffen, D. A., and Thompson, D. J. 1975, Ap. J., 199, 54. 
Black, J. H., and Fazio, G. G. 1973, Ap. J. (Letters), 185, L7. Brown, R., and Gould, R. J. 1970, Phys. Rev., D1, 2252.

Burton, W. B., Gordon, M. A., Bania, T. M., and Lockman, F. J. $1975, A p . J$. , in press.

Carruthers, G. R. 1970, Ap. J. (Letters), 161, L81.

Clark, B. G. 1965, Ap. J., 142, 1398.

Clark, G. W., Garmire, G. P., and Kraushaar, W. L. 1970, in Proc. IAU Symposium 37 on Non-Solar X- and $\gamma$-Ray Astronomy, ed. L. Gratton (Dordrecht: Reidel), p. 239.

Crasemann, B., Koblas, P. E., Wang, T., Birdseye, H. E., and Chen, M. H. 1974, Phys. Rev., A9, 1143.

Daltabuit, E., and Meyer, S. 1972, Astr. and Ap., 20, 415.

Daniel, R. R., and Stephens, S. A. 1975, Space Sci. Rev., $17,45$.

Dodds, D., Strong, A. W., and Wolfendale, A. W. 1975, M.N.R.A.S., 171, 569.

Dodds, D., Strong, A. W., Wolfendale, A. W., and Wdowczyk, J. 1974, Proc. ESLAB Symp. on Gamma-Ray Astronomy, Frascati (ESRO SP-106), p. 221.

Emerson, D. T. 1974, M.N.R.A.S., 169, 607.

Fichtel, C. E., Hartman, R. C., Kniffen, D. A., Thompson, D. J., Bignami, G. F., Ögelman, H., Özel, M. F., and Tümer, T. 1975, Ap. J., 198, 163.

FitzGerald, M. P. 1968, A.J., 73, 983.

Ginzburg, V. L., and Khazan, Ya. M. 1972, Ap. Letters, 12, 155.

Ginzburg, V. L., and Syrovatskii, S. I. 1963, Proiskhozhdeniye Kosmicheskikh Luchay (English Trans., Origin of Cosmic Rays [New York: Macmillan: 1964]).

Goldreich, P., and Kwan, J. 1974, Ap. J., 189, 441.

Gorenstein, P. 1975, Ap.J., 198, 95.

Gottesman, S. T., and Weliachew, L. 1975, Ap. J., 195, 23.

Guibert, J. 1974, Astr. and Ap., 30, 353.

Heiles, C., and Jenkins, E. B. 1975, submitted for publication.

Hollenbach, D. J., and Salpeter, E. E. 1971, Ap. J., 163, 155.

Hollenbach, D. J., Werner, M. W., and Salpeter, E. E. 1971, Ap. J., 163, 165

Ilovaisky, S. A., and Lequeux, J. 1972, Astr. and Ap., 20, 347.

Jackson, P. D., and Kellman, S. A. 1974, Ap. J., 190, 53.

Jenkins, E. B., and Savage, B. D. 1974, Ap. J., 187, 243.

Kaplan, I. G., and Markin, A. P. 1973, Soviet Phys.-JETP, 37, 216.

Kerr, F. J. 1969, Ann. Rev. Astr. and Ap., 7, 39.

Kerr, F. J., and Westerhout, G. 1965, in Galactic Structure, Vol. 5 of Stars and Stellar Systems, ed. A. Blaauw and M. Schmidt (Chicago: University of Chicago Press), p. 167.

Knapp, G. R., and Kerr, F. J. 1974, Astr. and Ap., 35, 361.

Kniffen, D. A., Hartman, R. C., Thompson, D. J., and Fichtel, C. E. 1973, Ap. J. (Letters), 186, L105.

Kodaira, K. 1974, Pub. Astr. Soc. Japan, 26, 255.

Kron, G. E., and Guetter, H. H. 1973, Pub. A.S.P., 85, 534

Lynds, B. T. 1962, Ap. J. Suppl., 7, 1.

McClure, R. D. 1969, Ap. J., 74, 50.
Mezger, P. G. 1970, in Proc. IAU Symposium 38, ed. W. Becker and G. Contopoulos (Dordrecht: Reidel), p. 107.

Parker, E. N. 1969, Space Sci. Rev., 9, 651.

Peimbert, M. 1968, Ap. J., 154, 33.

Price, R. M. 1974, Astr. and Ap., 33, 33.

Puget, J. L., and Stecker, F. W. 1974, Ap. J., 191, 323.

Roberts, W. W. 1970, in Proc. IAU Symposium No. 38, ed. W. Becker and G. Contopoulos (Dordrecht: Reidel), p. 415

Ryter, C. E., Cesarsky, C. J., and Audouze, J. 1975, Ap. J., 198, 103.

Sanders, R. H., and Prendergast, K. H. 1974, Ap. J., 188, 489.

Schlickeiser, R., and Thielheim, K. O. 1974, Astr. and Ap., 34, 167 .

Schmidt, M. 1965, in Galactic Structure, ed. A. Blaauw and M. Schmidt (Chicago: University of Chicago Press), p. 513. Scoville, N. Z., and Solomon, P. M. 1974, Ap.J. (Letters), 187, L67.

- 1975, ibid., 199, L105.

Scoville, N. Z., Solomon, P. M., and Jefferts, K. B. 1974, Ap. J. (Letters), 187, L63.

Searle, L. 1971, Ap. J., 168, 327

Shane, W. W. 1972, Astr. and Ap., 16, 118.

Solomon, P. M. 1973, Phys. Today, 26, 32

Solomon, P. M., and Stecker, F. W. 1974, Proc. ESLAB Symp. on the Context and Status of Gamma-Ray Astronomy, Frascati (ESRO SP-106), p. 253.

Solomon, P. M., and Wickramasinghe, N. C. 1969, Ap. J., 158, 449.

Spinrad, H., Liebert, J., Smith, H. E., Schweitzer, F., and Kuhi, L. 1971, Ap. J., 165, 17.

Spitzer, L., Drake, J. F., Jenkins, E. B., Morton, D. C., Rogerson, J. B., and York, D. G. 1973, Ap.J. (Letters), 181, L116.

Stecher, T. P., and Stecker, F. W. 1970, Nature, 226, 1234.

Stecker, F. W. 1969, Nature, 222, 865

ㄴ. 1970, Ap. and Space Sci., 6, 377. Corp.). 1971, Cosmic Gamma Rays (Baltimore: Mono Book

1973, Ap. J., 185, 499

- - 1975a, in Origin of Cosmic Rays, ed. J. L. Osborne and A. W. Wolfendale (Dordrecht: Reidel), p. 267

__ 1975b, Phys. Rev. Letters, 35, 188

Stecker, F. W., Puget, J. L., Strong, A. W., and Bredekamp, J. H. 1974, Ap. J. (Letters), 188, L59.

Strong, A. W. 1975, J. Phys. A, 8, 617.

Strong, A. W., Wdowczyk, J., and Wolfendale, A. W. 1973, in Gamma Ray Astrophysics, ed. F. W. Stecker and J. I. Trombka (NASA SP-339), p. 239.

Tucker, W., Kellogg, E., Gursky, H., Giacconi, R., and Tananbaum, H. 1973, Ap.J., 180, 715.

Webber, W. R. 1968, Australian J. Phys., 21, 845.

Westerhout, G. 1970, in Galactic Astronomy, ed. H. Y. Chiu and A. Muriel (New York: Gordon \& Breach).

F. W. Stecker: Theoretical Studies Group, NASA Goddard Space Flight Center, Greenbelt, MD 20771

P. M. Solomon: Department of Earth and Space Sciences, State University of New York, Stony Brook, NY 11794

N. Z. Scoville: Department of Physics and Astronomy, University of Massachusetts, Amherst, MA 01002

C. E. RYTER: Laboratory for High Energy Astrophysics, NASA Goddard Space Flight Center, Greenbelt, MD 20771 